WIENER SLAVISTISCHES JAHRBUCH, Band 54/2008, 63-76

(C) 2008 by Österreichische Akademie der Wissenschaften, Wien

B E R N A R D A K A T U Š I Ć

\title{
August Šenoas Roman Zlatarevo zlato als Variante des Kunstmärchens
}

Als Forschungsobjekt der Literaturtheorie stellt sich der Märchenbegriff hinsichtlich der Frage nach seinen Wesensmerkmalen als ein vielschichtiger, bisweilen mit Widersprüchen behafteter Gegenstand dar. Wie kaum ein anderes literarisches Genre auf ein relativ beschränktes Formenrepertoire verwiesen, verfügt das Märchen gleichzeitig über die Fähigkeit, gesellschaftliche und ästhetische Veränderungen in sich aufzunehmen und ihnen nach Maßgabe seiner Mittel Ausdruck zu verleihen. Strenge Gesetzmäßigkeit einerseits und große Variabilität anderseits sind die Bestandteile des einem ständigen Wandlungsprozess unterworfenen, normativ wirkenden poetologischen Musters, das seine Struktur kennzeichnet. Präziser formuliert, tritt das Märchen - trotz seines scheinbar äußerst schablonenhaften Aufbaus - nicht in einer starren Form auf, seine Eigentümlichkeit besteht gerade darin, dass es seine Gestalt ,amöbenhaft wechseln kann“ (Karlinger 1973: VII).

In diesem Sinn ist die Wahrnehmung eines Produkts der (dichterischen) Phantasie als Märchen weder an ein bestimmtes Genre noch an ein bestimmtes Medium, weder an eine konkrete Epoche noch an ein besonderes Rezipientenprofil gebunden. Wesentlich mit dem Moment des Archaisch-Mythischen behaftet, ist es zugleich eine äußerst flexible ästhetische Ausdrucksform, die mit der Literaturgeschichte und ihren epochalen Veränderungen gleichsam mitwächst und sich mühelos an die Ansichten und den Geschmack des jeweiligen Zeitgeists anpassen kann (vgl. Biti 2001: 531). Primär handelt es sich beim Märchen um eine epische Kunstform - es tritt vornehmlich in Gestalt einer kurzen oder längeren Erzählung, aber auch eines Novellenzyklus oder Romans auf -, seine Sujets finden sich aber ebenso als Verse gestaltet oder in dramatischer Form - als Theaterstück, als Film oder als Performance - 
aufbereitet. ${ }^{1}$ Die Überlieferung erfolgt sowohl auf mündlichem als auch auf schriftlichem Weg, was heute selbstverständlich die Abrufbarkeit in elektronischer Form einschließt. ${ }^{2}$ Kurzum: Als eines der ältesten und am weitesten verbreiteten Kulturgüter der Menschheit ist das Märchen „ebenso urzeitlich wie unzeitlich, d. h. immanent und konstant im Wesen des Menschen veranlagt“" (vgl. Ranke 1978: 1-4).

Vor diesem Hintergrund hat sich die Wissenschaft des Märchens unter den verschiedensten methodologischen Aspekten - vom ethnologischen über den philologischen, religiösen, anthropologischen, soziologischen, psychologischen, psychiatrischen und pädagogischen bis hin zum literaturtheoretischen - angenommen, und doch liegt bislang, ungeachtet der mittlerweile schier unüberschaubaren Menge an Fachliteratur, keine endgültige Begriffsdefinition vor. Als einer der Hauptgründe, warum „wir zwar bei der Fachwelt wohl eine große Fülle von Vorstellungen, die sich freilich nicht selten widersprechen, finden, aber daraus keinen einheitlichen und sich begrifflich fassbaren Eindruck gewinnen“ (Karlinger 1973: VII), lässt sich die Tatsache nennen, dass das Märchen einerseits ein ahistorisches Faktum ist, das durch synchrone Merkmale, wie die Variabilität seiner Gesetzmäßigkeiten, seine ungeklärte Genese und die schwer nachvollziehbaren Wege seiner Verbreitung gekennzeichnet, anderseits aber historisch bedingt und somit nur in einem konkreten diachronen Kontext bestimmbar ist. Mit anderen Worten: Obwohl in allen Kulturen und Epochen gegenwärtig, ist das Märchen auch ein Begriff, der nicht ,aus sich selbst" (vgl. Jolles 1972: 231) entstanden ist, sondern, wie jede theoretische Bestimmung, ein Konstrukt, das sowohl von einem bestimmten historisch-gesellschaftli-

${ }^{1}$ So gelten im literaturwissenschaftlichen Diskurs als Märchen nicht nur mündlich überlieferte Volkserzählungen und deren mehr oder weniger bearbeitete und autorisierte schriftliche Fixierung, wie die Märchen von Perrault, den Brüdern Grimm und Andersen, sondern auch hoch stilisierte, allegorisch-symbolische Geschichten, wie die Märchen Goethes und Wildes, die phantastischen Erzählungen E. T. A Hoffmanns und Kafkas oder die realistischen Werke aus der Feder von Dickens, Thackeray und Keller. Als Märchen qualifiziert und interpretiert werden des Weiteren nicht nur kürzere Erzählformen, sondern auch umfangreiche Novellenzyklen und ebenso Romane verschiedensten Umfangs, wie Die Erzählungen aus Tausendundeiner Nacht, Straparolas Die ergötzlichen Nächte, Basiles Das Pentameron, Carrolls Alice im Wunderland, Collodis Pinocchio, Endes Märchenromane, Rowlings Harry Potter und der Stein der Weisen, Funkes Tintenherz etc. Von den in Versform bzw. als Theaterstück gestalteten Märchensujets sind etwa Puškins Versmärchen und die Dramen von Maeterlinck zu nennen. $\mathrm{Zu}$ einer für die Gattung repräsentativen Auswahl von Märchentexten vgl. Crnković 1990, Klotz 2002 und Neuhaus 2005.

2 Im Zeitalter der neuen Medien (Film, Radio, Internet) hat das Märchen seinen variablen Charakter wie auch seine Anpassungsfähigkeit noch einmal bestätigt. Die anhaltende Popularität der Gattung lässt sich nicht nur anhand ständig nachgedruckter Buchauflagen und zahlreicher Verfilmungen ,klassischer“" Märchentexte dokumentieren, sondern auch durch diverse Internetinitiativen mit ihren online angebotenen Märchenspielen und dem online Lesen, Erfinden und Weitererzählen von Märchen, wie auch insbesondere durch die Existenz von Märchengesellschaften, die sich der Verbreitung des Märchens auf institutioneller Ebene annehmen. Für nähere Informationen vgl. http://www.maerchen-emg.de (abgerufen am 17.09.07). 
chem Zusammenhang, als auch von dem ethnischen, kulturellen und individuellen Blickwinkel, aus dem es betrachtet wird, abhängt.

So verstanden ist das Märchen in der abendländischen Kultur ein relativ neuer literaturtheoretischer Begriff, den man erst im 19. Jahrhundert systematisch zu untersuchen begann. Erst ab dieser Zeit wurden Theorien nicht nur seine Gestalt, sondern auch den Zeitpunkt seiner Entstehung und seine Herkunft betreffend, formuliert. Und wenngleich die damals vorgelegte Definition ${ }^{3}$ als archaische, mündlich überlieferte Erzählung, die sich von anderen Formen der Volksdichtung, allen voran der Sage, der Legende und dem Schwank, durch wundersame Inhalte und „naive“ Moral unterscheidet, in gewisser Weise noch heute Gültigkeit hat, lässt sich seine Geschichte im europäischen literaturtheoretischen Kontext, je nachdem, ob bei seiner Erforschung der Akzent auf der Konvergenz der Motive, seiner Struktur oder auf der Veränderlichkeit seiner Gesetzmäßigkeiten liegt, in drei Etappen teilen. ${ }^{4}$

In der ersten Etappe, die mit dem Aufkommen romantischer Tendenzen einsetzte und ungefähr bis in die 30er Jahre des 20. Jahrhunderts andauerte, wurden primär die $\mathrm{zu}$ archaischen Zeiten in menschlichen Gemeinschaften geltenden Normen, Vorstellungen und Glaubensinhalte, die sich durch orale Überlieferung in mehr oder weniger „transparenter“ Form in Märchenmotiven erhalten haben, untersucht (vgl. Biti 2001: 530). In diesem Zusammenhang rückten Fragen nach Herkunft und Alter des Märchens, sowie nach den Wegen der Verbreitung seiner Motive in den Mittelpunkt der Forschung.

In der zweiten, mit den wissenschaftlichen Debatten der 30er und 40er Jahre des 20. Jahrhunderts beginnenden Etappe, setzte sich dagegen die Ansicht durch, dass die Wesensmerkmale des Märchens nicht auf dem Wege der stofflichen Zerlegung in Motive, sondern in der Art und Weise der Motivverwendung und -verknüpfung zu finden sind (vgl. Bausinger 2004: 178). Demgemäß werden in dieser Phase die wichtigsten Parameter der Märchenform nicht mehr auf der inhaltlichen Ebene, sondern in strukturellen Merkmalen, wie Komposition und Syntax, sowie im Grad der Stilisiertheit gesucht. Die - vor allem von Propp und Lüthi praktizierte - theoretische Fokussierung auf die formalen Gesetzmäßigkeiten des Genres markiert die definitive Änderung des methodischen Ansatzes, das Ersetzen der Frage, wo und wie das Märchen entstanden ist, durch jene, was das Märchen an sich ist. Anders als die bis dahin dominierende, vorwiegend auf historisch-genetischer Basis betriebene Märchenforschung beabsichtigte Propp in seiner Morphologie des Märchens (1928),

${ }^{3}$ Bis heute wird das Bild nicht nur des deutschen, sondern des abendländischen Märchens allgemein vor allem durch die Kinder- und Hausmärchen (1812-15) der Brüder Grimm geprägt; in der Literaturwissenschaft ist das Märchen daher oft auch als „Grimm-Gattung“ bezeichnet worden (vgl. dazu Jolles 1972: 219, Brackert 1994: 10, Bausinger 2004: 176).

4 Es ist hier nicht der Ort, die äußerst komplexe Geschichte des Märchenbegriffs aus einem fachübergreifenden Blickwinkel darzustellen. Eine knappe Entwicklung des Märchenbegriffs aus der literaturwissenschaftlichen Perspektive möge genügen, um zu zeigen, wie sich der Märchenbegriff im Abendland konstituiert und konstruiert hat. 
„ein strukturelles Grundprinzip“ (vgl. Propp 1975: 13) aufzubauen, auf das sich sämtliche Märchenformen zurückführen lassen könnten. ${ }^{5}$ Während Propp die Grundform des Märchens also an seiner morphologischen Struktur festmacht, siedelt Lüthi seine konstitutiven Elemente auf der Stilebene an. In seinem Buch Das europäische Volksmärchen (1947) geht er zudem davon aus, dass das Geheimnis des Märchens nicht in den Motiven, die es verwendet, sondern in der Art und Weise, wie es das tut bzw. „,in seiner Form, die nicht aus dem Stoff erwächst, sondern aus sich selber lebt“ (vgl. Lüthi 1997: 6), ruht. In diesem Zusammenhang operiert er mit fünf stilistischen Grundkategorien - Eindimensionalität, Flächenhaftigkeit, abstrakter Stil, Isolation und Allverbundenheit sowie Welthaftigkeit -, die die Basis für die phänomenologische Analyse der Märchenform bilden sollten.

Die von Lüthi entwickelten, auf dem Stil beruhenden Märchenmerkmale und ebenso Propps handlungsbedingte „Funktionen der Akteure“ mögen eine unumgehbare Konstante auch aller künftigen Märchenforschung darstellen - ihr explizites Anliegen, den Begriff dessen, was „das Märchen an sich“ (vgl. Lüthi 1997: 6 und Propp 1975: 13) ist, zu liefern, vermochten beide Autoren damit gleichwohl nicht zu verwirklichen. Bestenfalls treffen ihre Erkenntnisse auf einen einzelnen Märchentypus, nämlich das Volksmärchen, zu, das im 18. Jahrhundert in weiten Teilen Europas mündliche Verbreitung fand und zumeist im 19. Jahrhundert schriftlich fixiert wurde. $^{6}$

Im Zuge der in den letzen vierzig Jahren des 20. Jahrhunderts (vgl. Bogatyrev Jakobson 1972, Ranke 1978, Bausinger 2004) durchgeführten wissenschaftlichen

${ }^{5}$ Der Ausgangspunkt einer solch umfassenden morphologischen Strukturbeschreibung des Märchens war für Propp die Erkenntnis in Bezug auf die Priorität der Ereignisse. Er machte darauf aufmerksam, dass die Bestandteile eines Märchens auf ein anderes übertragen werden können, dass z. B. die Gestalt der Hexe Baba-Jaga in den unterschiedlichsten Märchen auftreten kann, ohne dass sich der Gang der Erzählung verändert. Entscheidend für die Bestimmung eines Märchens ist für ihn in diesem Sinne nicht die Hexenfigur, sondern das strukturelle Gefüge und die darin ablaufenden Handlungen, die er als ,Funktionen der Akteure" bezeichnet. Propp operiert mit 31 solchen Funktionen: zeitweilige Entfernung, Verbot, Verletzung des Verbots, Verrat, Betrugsmanöver, Mithilfe, Schädigung, Vermittlung, einsetzende Gegenhandlung, Abreise, Funktion des Schenkers, Reaktion des Helden, Empfang eines Zaubermittels, Raumvermittlung (Wegweisung), Kampf, Kennzeichnung (Markierung), Sieg, Liquidierung (Aufhebung des Unglücks oder Mangels), Rückkehr, Verfolgung, Rettung, unerkannte Ankunft, unrechtmäßige Ansprüche, Prüfung (schwere Aufgabe), Lösung, Erkennung, Überführung (Entlarvung), Transfiguration, Strafe (Bestrafung), Hochzeit und Thronbesteigung (vgl. Propp 1975: 31-67).

${ }^{6}$ Während Lüthi seine Forschung auf die europäischen Volksmärchen konzentriert (vgl. Lüthi 1997: 6), beruhen die von Propp vorgenommenen systematischen Beschreibungen ausschließlich auf den so genannten Zaubermärchen oder „Märchen im eigentlichen Sinne des Wortes“ (vgl. Propp 1975: 9). Bei ihren Untersuchungen gehen beide Autoren von der These aus, dass das Märchen eine statische Urform darstellt, und aus diesem Grund verfolgen sie nicht seine Veränderungen in der Geschichte, sondern ziehen die Überlieferungen, die ihnen durch historische Vermittlung, in mehr oder weniger autorisierter und bereits bearbeiteter Schriftform zugänglich waren, als über jeden Zweifel erhabene Zeugen einer langen Kontinuität heran. 
Untersuchungen gelangte man schließlich zur Auffassung, dass es sich beim Märchen nicht, wie von Propp und Lüthi vermutet, um ein in prähistorischer Zeit entstandenes mythisches Volksgut handelt, das in unveränderter Gestalt mündlich von Generation zu Generation überliefert wird, sondern eine stets auf neue Weise tradierte literarische Form, die in der Lage ist, unterschiedlichen historischen und kulturellen Entwicklungen sowie den unterschiedlichen Erwartungen ihrer Rezipienten Rechnung zu tragen und sich zudem zur Vermittlung über verschiedenste mediale Formen eignet. Während die bis dahin betriebene Forschung das Märchen als eine statisch konservierte Form betrachtete, rückte nunmehr die „Veränderlichkeit der Märchendiskursivität" (vgl. Biti 2001: 531) in den Mittelpunkt der wissenschaftlichen Debatte, und die Untersuchung galt einerseits den Veränderungen im Überlieferungsprozess, dem Kontext des Erzählers, seinem Publikum sowie dessen spezifischem gesellschaftlichen Umfeld ${ }^{7}$ und anderseits der Wechselwirkung zwischen schriftlicher und mündlicher Überlieferung. Hatte sich das theoretische Denken lange Zeit auf die Gegensatzpaare Oralität / Schriftlichkeit, Natur / Kunst, Anonymität / Autorschaft, Naivität / Ernsthaftigkeit sowie Zeitlosigkeit / Geschichte konzentriert, die in der Regel als die konstitutiven Elemente des Märchens galten, wurde in dieser Forschungsphase eine solche klare Gegensätzlichkeit mehr und mehr in Frage gestellt. Folglich wandte man sich vermehrt dem Kunstmärchen ${ }^{8} \mathrm{zu}-$ nicht mehr im Sinne eines Gegenbegriffs zum Volksmärchen, sondern einer ,uneigenständigen literarischen Gattung“" (vgl. Klotz 2002: II) ${ }^{9}$, die sich auf das Volksmärchen als Orien-

${ }^{7}$ Diesbezüglich wird in neueren wissenschaftlichen Untersuchungen darauf aufmerksam gemacht, wie in der Märchenerzähltradition in Gegenden, in denen das lebendige mündliche Erzählen von Märchen bis in die Gegenwart reicht, immer wieder höchst moderne Züge und Requisiten auftauchen: Der König telefoniert mit seiner Frau; der Märchenheld reist, um schnell in ein anderes Land zu kommen, mit dem Flugzeug; die sieben Zwerge stimmen demokratisch ab, was mit Schneewittchen geschehen soll (vgl. Bausinger 2004: 175).

8 Auch wenn der Begriff „Kunstmärchen“ als Bezeichnung für eine literarische Form auf die Brüder Grimm zurückgeht, wird er erst ab dieser Phase nicht mehr als ein Gegenbegriff zum Volksmärchen aufgefasst, sondern als eine literarische Form, die nicht nur in Bezug auf das Volksmärchen, sondern auch aus sich selbst und folglich aus ihren verschiedenen Entwicklungsstufen zu bestimmen ist. Besonders treffend wurde dieser Umstand von Tismar zum Ausdruck gebracht, der das Kunstmärchen folgendermaßen definierte: „Das Kunstmärchen stellt den Versuch dar, das ahistorische Volksmärchen in der jeweiligen historischen Gegenwart auszulegen. Doch nicht so, daß das Kunstmärchen jeweils von neuem spontan das Volksmärchen-Muster aktualisiert. Es bildet, indem es sich an den bereits vorhandenen Beispielen solcher Interpretation orientiert, eine eigene Tradition aus, auf die es sich berufen kann" (Tismar 1981: 1).

9 Klotz fasst das Märchen als eine ,uneigenständige literarische Gattung“ auf, in erster Linie aufgrund dessen, weil er in seiner Märchendefinition vom Erwartungshorizont und von den Vorstellungen, die das Märchen bei den Autoren und Rezipienten hervorruft, ausgeht. „Kunstmärchen [...] sind literarische, geschichtlich und individuell geprägte $\mathrm{Ab}$ wandlungen der außerliterarischen, geschichtlich unbestimmten, anonymen Gattung Volksmärchen durch namhafte Autoren. Diese Autoren rufen also bei ihrer Leserschaft sowohl das vertraute Muster jener markanten Erzählform ab wie auch die eher diffusen 
tierungsmuster bezieht, wenn auch in von Fall zu Fall unterschiedlicher Weise und in unterschiedlichem Maß (vgl. Tismar 1981: 1, Bausinger 2004: 174, Brackert 1994: 10). Aus dieser Perspektive betrachtet, stellte sich das Kunstmärchen als eine traditionelle Form dar, die sich leicht „für neue Erfahrungen öffnet“ (vgl. Brackert 1994: 15), als ein Genre, das aufgrund der reichen, vom Volksmärchen bezogenen Mitgift über ,größere Möglichkeiten als viele ältere und jüngere literarische Gattungen“" (vgl. Klotz 2002: 6) verfügt.

Im Vergleich zu den „großen“ europäischen Literaturen, in denen das Märchen demnach nicht mehr nur als eine „volkstümliche“, mündlich überlieferte „einfache Form“ (Jolles 1972) figuriert, sondern gleichzeitig als eine höchst produktive literarische Gattung angesehen wird (vgl. Petzold 1981, Brackert 1994, Klotz 2002, Neuhaus 2005, Wührl 2003), begegnet die kroatische Literatur dem Märchen mit großem Misstrauen, das sich nicht nur in der geringen Quantität einschlägiger Produktionen, sondern auch in deren Stellenwert innerhalb der literarischen Ausdrucksmöglichkeiten manifestiert. Wann immer die jüngere kroatische Literaturwissenschaft sich dieser Gattung widmet, meint sie damit zumeist noch immer entweder eine archaische Form der Volksdichtung, die sich von anderen mündlich überlieferten Erzählungen (Sage, Legende, Schwank, etc.) durch irrationale Inhalte, einen hohen Stilisierungsrad und naive Moral unterscheidet (vgl. Biti 1981, Bošković-Stulli 1982, Kekez 1983, Solar 2004), oder aber eine Abteilung der Kinderliteratur, die auf Grund ihres phantastischen Weltbildes, ihrer klaren Struktur und einfachen Sprache sowie ihrer durchsichtigen, auf den Triumph des „Guten“ abzielende Botschaft nur die Bedürfnisse von Adressaten mit geringem Reflexionsvermögen befriedigen kann (vgl. Crnković 1990, Težak 1997, Vrkić 1997, Matičević 1997).

Insofern hat sich die kroatische Literaturwissenschaft dem Märchen bislang fast ausschließlich entweder aus einer volkskundlichen oder einer literaturdidaktischen Perspektive genähert. ${ }^{10}$ Ein solcher, offenkundig unzulänglicher Zugang zur Märchenform hatte einerseits zur Folge, dass auf wichtige Kontextbildungen und überhaupt darauf, Märchen auf ihre spezifische literarische oder ästhetische Qualität hin zu befragen, verzichtet wurde und anderseits, dass viele Texte aus dem zentralen Korpus der neueren kroatischen Literatur, die klar erkennbar Märchenelemente aufweisen, aber aufgrund ihres komplexen Aufbaus nicht als Kinderliteratur geeig-

Vorstellungen, die man ihr, im Laufe der Zeit, abgewonnen hat. Die Summe der Kunstmärchen ergibt ihre eigenartige, aber zunächst uneigenständige literarische Gattung. Und ihre fortschreitende Abfolge gibt die Geschichte der Gattung andeutungsweise zu erkennen“ (Klotz 2002: II).

${ }^{10}$ Nicht nur in der kroatischen, auch in anderen „kleinen“ Literaturen wird das Märchen noch immer primär aus einer literaturdidaktischen Perspektive untersucht. Es finden sich daher im Kontext der südslawischen Literaturen nur sehr wenige Arbeiten, die das Problem der „Transposition des Märchens in die Kunstliteratur“ (vgl. Drndarski 1978: 17) behandeln; vgl. dazu auch Šutić 1978, Žmegač 1997, Katušić 2006. 
net sind, außerhalb des Interessenshorizonts der Märchenforschung geblieben sind. ${ }^{11}$ Diese widmete sich der ästhetischen Bedeutung des Märchens in der kroatischen schriftlichen Literatur unter lediglich zwei Aspekten und blieb dementsprechend reduziert. $^{12}$

Wird jedoch das Märchen nicht als statische, ausschließlich der Volks- oder Kinderliteratur vorbehaltene Ausdrucksform betrachtet, sondern als eine Gattung, die sich in jeder historischen Etappe wie auch in jeden Nationalliteratur immer anders konstituiert, erweist sie sich auch in der kroatischen Literatur als eine fruchtbare Ausdrucksweise, die sich ihren Weg von der Romantik über den Realismus und die Moderne bis hin zur Postmoderne zu bahnen vermocht hat. Im Folgenden wird der Versuch unternommen, die durch die spezifischen gesellschaftlichen und historischen Verhältnisse geprägte Entwicklung des Märchens in der kroatischen Literatur des 19. Jahrhunderts anhand der Analyse des ersten historischen Romans Zlatarevo zlato [Des Goldschmieds Gold] (1871) von August Šenoa, der gemeinhin als der

${ }^{11} \mathrm{Zu}$ diesen Texten zählen beispielsweise Šenoas Erzählverse Kugina kuća [Das Pesthaus] (1869) und Kameni svatovi [Die versteinerten Hochzeitsgäste] (1869), Šops Prosasammlung Tajanstvena prela [Das geheimnisvolle Spinnen] (1943), Galović' Novelle Začarano ogledalo [Der verzauberte Spiegel] (1913), Matoš' symbolistische Novellen Camao (1900), Cvijet s raskršća [Blume am Kreuzweg] (1909), Balkon [Der Balkon] (1909), etc. Insbesondere die jüngste literarische Produktion weist eine Fülle von Texten auf, die explizit mit der Märchenform arbeiten, wie beispielsweise Slamnigs Botanička bajka [Das botanische Märchen] (1980), Ugrešić' Život je bajka [Das Leben ist ein Märchen] (1983), Budišas Princeza [Die Prinzessin] (1984), Petković' Priče iz davnine [Erzählungen aus der Vorzeit] (1989), Rešickis Bajka [Märchen] (1993), Dragojević' Bajka o vratima [Ein Märchen von der Tür] (1994), Ferić' Tužna bajka o Clari Schumann i braći Grimm [Trauriges Märchen von Clara Schumann und den Gebrüdern Grimm] (2000), etc. Dass ein solcher Boom des Märchengenres nicht nur ein Phänomen der rezenten kroatischen Literatur, sondern eine genuin postmoderne Erscheinung ist, die die Grenzen einer Nationalliteratur weit überschreitet, lässt sich leicht durch den Verweis auf die Werke von Calvino über Márquez, G. Roth, Petruševskaja, Ćosić, Kiš, Pavić etc. belegen.

12 Die Frage, warum in der kroatischen Literatur der Märchenform eine solche, geradezu suspekte, Rolle zugewiesen wird, lässt sich nicht eindeutig beantworten, doch es können zumindest zwei Gründe vermutet werden, die eine derartige Zurückhaltung in diesem Forschungsbereich erklären könnten. 1. Märchentradition. Da die kroatische Literatur lange Zeit ganz im Zeichen moralischer und religiöser Unterweisung stand, war die Aufzeichnung mündlich überlieferter Erzählungen und Gedichte - und insbesondere von Märchen als „,erfundene“ Erzählungen, die nicht direkt belehren - im Vergleich zu anderen europäischen Literaturen weit zurückgeblieben (vgl. dazu mehr Bošković-Stulli 1975: 274-281). 2. Auffassung des Märchenbegriffs. Auch wenn der Märchenbegriff als Ausdruck für eine Erzählung über unglaubliche und wundersame Geschehnisse im Kroatischen bereits im 19. Jahrhundert in Gebrauch war, wurde er auf Grund dessen, dass er von anderen Gattungen der Volksdichtung nicht ausreichend differenziert wurde, im literaturwissenschaftlichen Diskurs bis in die zweite Hälfte des 20. Jahrhunderts nur sehr selten verwendet. Bis in die jüngste Zeit benutzte man sowohl für die ,wundersamen“ Geschichten als auch für die im Volk kursierenden Erzählungen allgemein viel öfter als den Begriff Märchen den Begriff narodna priča [Volkserzählung]. So trägt beispielweise die erste Kunstmärchensammlung der kroatischen Literatur, die Erzählsammlung von Ivana Brlić Mažuranić den Titel Priče iz davnine (1916) [dt. Aus Urvaterzeiten, 1933]. 
erste ästhetisch relevante Roman der neueren kroatischen Literatur betrachtet wird, aus eben diesem Blickwinkel zu beleuchten.

Der in der europäischen Romantik sich vollziehende Übergang von der mündlichen Überlieferung zur schriftlichen Fixierung, die Abwendung vom kollektiven und die Hinwendung zum individuellen Rezipienten sowie die entsprechende sprachliche Adaptierung leitete nicht nur eine neue historische Etappe der Märchenentwicklung ein, sondern bewirkte auch dessen Aufwertung zur wichtigsten literarischen Ausdrucksform des romantischen Weltbildes, zu einem paradigmatischen, übergreifenden Prinzip, das für jede Form von Poesie Gültigkeit haben sollte (vgl. Lüthi 1977: 5, Žmegač 1994: 263, Klotz 2002: 138-139) ${ }^{13}$. Obwohl in der kroatischen Literatur das Märchen zu dieser Zeit im Schatten von Literaturformen, wie der sentimentalen Liebesgeschichte, der Heiducken- und Türkennovelle oder dem Heldenepos, steht, die - im Einklang mit den damaligen ästhetischen Auffassungen fähig waren, dem neu erwachten Geist einer unterdrückten Nation eine konkretere und machtvollere Stimme zu verleihen, lässt sich seine Präsenz in Gestalt einer nicht explizit reflektierten Gattung ebenso wenig negieren wie eine eigene latente ästhetische und ethische Grundlage. Mit Blick auf andere europäische Länder, in denen mit dem Ausklingen romantischer Tendenzen das Interesse an der Form des Märchens merklich abnahm, scheint es sogar gerechtfertigt, nicht nur die gesamte Tradition der kroatischen Romantik, sondern auch viele realistisch konzipierte Werke dieser Zeit aufgrund ihres unzureichenden kritischen Potentials und der in Zusammenhang damit oft hervorgehobenen romantischen Gefühlsseligkeit zur ästhetischen Konzeption des Märchens in Beziehung zu setzen. In Hinblick darauf, dass die kroatische Literatur bis zum Beginn des 20. Jahrhunderts durch einen eigentümlichen, undifferenzierten Synkretismus, der anderen europäischen Literaturen fremd war, durch ein Gemenge von konventionellen romantischen und realistischen Elementen und durch das Spannungsverhältnis zwischen programmatischen Vorhaben und konkreter ästhetischer Realisierung charakterisiert ist, bietet sich aus heutiger Perspektive die dem Märchen eigene Diskursivität als adäquates Instrumentarium für das Lesen der gesamten kroatischen Literatur des 19. Jahrhunderts an.

In der Literaturkritik herrscht weitgehend Einigkeit darüber, dass Šenoa, indem er die kroatische Literatur nicht nur endgültig vom Dilettantismus befreite (vgl. Barac 1977: 153, Šicel 1993: 12, Nemec 1994: 79), sondern auch zwei bereits das 19. Jahrhundert dominierende, an sich konträre literarische Strömungen - die Romantik und den Realismus - in sie einführte, die Grundlage dafür geschaffen hat, dass sich die neuere kroatische Literatur überhaupt konstituieren konnte und ihm insofern in

${ }^{13}$ Insbesondere in der deutschen Romantik, von Novalis über Tieck, Fouqué, Brentano, Eichendorff bis hin zu Hoffmann, war das Märchen eine der zentralen literarischen Gattungen. 
ihr eine zentrale Stellung zukommt. Vor allem in seinem vielfach zitierten programmatischen Artikel Naša književnost [Unsere Literatur] (1865) legte er sein bekanntes realistisches und engagiertes literarisches Konzept dar, mit dem er den literarischen Diskurs unter dem Gesichtspunkt der Reinheit und Klarheit der Darstellung kodifizierte: Demzufolge sind dessen konstituierende Momente das Maß, die Ordnung, der Einklang, die Chronologie und das Gleichgewicht aller zum Ausdruck gebrachten Formen (vgl. Lasić 1965: 35-61), die sowohl für die Generation der Romanschriftsteller zu Šenoas Zeit (Tomić, Kumičić), als auch für die erste und zweite Generation der kroatischen Realisten (Kozarec, Novak, Kovačić, Đalski) ein maßgeblicher Orientierungspunkt werden sollten. Durch die Ästhetik ,des Guten und des Fortschritts", den apodiktisch formulierten Imperativ, dass das gesamte literarische Schaffen sich in den Dienst der nationalen Sache zu stellen, den Volksgeist zu stärken und der Bildung und Aufklärung des Volkes zu dienen habe (vgl. Šicel 1972: 80-81), hat Šenoa eine Prosaformel vorgelegt, die für alle nachfolgenden Romanciers und Diskurse Verbindlichkeit haben sollte, er definierte sozusagen den Nullpunkt des kroatischen Romans, eine Norm, die assimiliert werden musste, um überwunden zu werden (vgl. Nemec 1994: 99).

Šenoas Roman Zlatarevo zlato - eine „Historička pripovijest 16. vijeka“ [Historische Geschichte aus dem 16. Jahrhundert], wie der Untertitel lautet - stellt einen seiner ersten Versuche dar, die Grundsätze der von ihm dahingehend interpretierten realistischen Kunstkonzeption auch in seinen literarischen Werken zu verwirklichen. Wie dem zu Beginn des Werks an den Leser gerichteten Vermerk zu entnehmen ist, verfolgt der Autor darin die Absicht, ein konkretes historisches Ereignis aus der kroatischen Geschichte, ,malenu sliku burne naše davnine“ [ein kleines Bild aus unserer stürmischen Vergangenheit], (Šenoa 1997: 1) - den Streit der Zagreber Bürger mit dem Adeligen Gregorijanec und dessen Anhängern um die Zagreber Vorstadt Medvedgrad - „detailliert und wahrheitsgetreu“ darzustellen.

Gledao sam da bude to vjerna prilika onog vremena. Tko mari uvjeriti se o tom, pročitaj tumač ovoj knjizi i vidjet će da se je sve što evo pripovijedam s veće strane uistinu zbilo, da su skoro sva lica mojeg djelca uistinu živjela i za života tako radila kao što se tude prikazuje. (Šenoa 1997: 1)

[Ich habe versucht, ein wahrheitsgetreues Abbild dieser Zeit zu geben. Wer sich davon überzeugen will, lese den Anhang dieses Buches, und er wird sehen, dass fast alles, was hier erzählt wird, auch tatsächlich geschehen ist, dass fast alle Personen meines Büchleins tatsächlich gelebt und in ihrem Leben genau so gehandelt haben, wie es hier dargestellt ist.] ${ }^{14}$

In der Tat muten die einzelnen Erzählsegmente - ganz in Übereinstimmung mit den Bestrebungen des Autors - höchst authentisch an, etwa das Treiben der histo-

14 Dass Šenoas Roman auf Grundlage eines intensiven Quellenstudiums und der Auseinandersetzung mit konkreten historischen Daten entstanden ist, geht aus dem Buchanhang hervor (vgl. Šenoa 1997: 231). 
risch verbürgten Persönlichkeiten ${ }^{15}$, die Veranschaulichung der Sitten und Lebensgewohnheiten von Bürgertum und Adel, die Darlegung der Pflichten und Rechte der damaligen Freistadt Zagreb, die im Detail wiedergegebenen politischen Auseinandersetzungen zwischen dem kroatischen Bürgertum und Österreich und Ungarn, ebenso wie das Verhalten der kroatischen Adeligen und Bürger von Zagreb auf Landtagssitzungen, bei Gerichtsprozessen und in Bürgerratsversammlungen, etc. Der Gesamteindruck jedoch, der sich bei der Lektüre ergibt, lässt sich weder als unmittelbare, objektive Wiedergabe realer Geschehnisse, noch als kritisch-analytische Schilderung der damaligen gesellschaftspolitischen Situation charakterisieren. Vielmehr wähnt sich der Leser bald von einer gleichsam märchenhaft-mythischen Atmosphäre umgeben, fühlt sich in eine wenn nicht unzeitliche, dann doch längst vergangene Epoche versetzt, in der die gesellschaftliche Ordnung ganz im Zeichen von Liebe und Gerechtigkeit stand und Harmonie zwischen den Menschen herrschte. Anders ausgedrückt, thematisiert der Roman - ungeachtet dessen, dass die Handlung von realen Personen getragen wird und ungeachtet ihrer konkreten raumzeitlichen Verortung - eine ,wirklichkeitsferne Welt", in der die Vielschichtigkeit und Komplexität erfahrbarer Realität auf eigentümliche Art und Weise verzaubert wirken; damit stellt er eine poetische Kreation völlig eigenen Gepräges dar (vgl. Lüthi 1997: 25).

Entgegen seiner programmatischen Beteuerung, ,wahrheits- und tatsachengetreu“ berichten zu wollen, verleiht Šenoa seinem Werk mittels der Einführung übermenschlicher Wirkungskräfte und überhaupt einer irrationalen Komponente eine klar umrissene, einem Ideal verpflichtete, moralische Funktion, für die die Schilderung der konkreten geschichtlichen Situation lediglich den Hintergrund abgibt. Eine Besonderheit der Poetik des Autors besteht demgemäß darin, dass er - unter Umgehung der propagierten realistischen Tendenz - unbewusst mit der poetischen Kategorie des Wundersamen und damit der Märchenstruktur arbeitet, insbesondere, wenn es um die Entfaltung des Plots und die Gestaltung der Figuren geht.

Ganz im Geist einer der Romantik verhafteten Hyperbolisierung des Gefühls bildet die dynamische Entfaltung des Plots den Schlüsselfaktor in Šenoas narrativer Konzeption. Wie im Märchen sind auch in diesem Geschichtsroman alle strukturellen Elemente der auf den Triumph des Guten über das Böse abzielenden Handlung untergeordnet. Sowohl Eigenschaften und Gefühle der agierenden Personen als auch die Erzählthemen drücken sich in diesem fiktiven Gebilde in erster Linie in der Handlung aus. Ungeachtet dessen, dass die narrativen Sequenzen des Textes wesentlich länger und komplexer verbunden sind als es die formalen Regeln des klassischen Märchens erlauben, drängt sich bei der Auslegung des Sujets aufgrund der gleichzeitigen „Isolation und Allverbundenheit“ (vgl. Lüthi 1997: 37) unmittelbar

15 Unter anderen sind Antun Vramec, der kroatische Chronist und der Zagreber Chorherr, Đuro Drašković, der Zagreber Bischof und der kroatische Banus, Angehöriger der bekannten kroatischen Adelsfamilien Frankopan und Zrinjski sowie der deutsche General Herbert Auersperg in die fiktive Handlung verwoben. 
die Assoziation mit der narrativen Kodifikation der Märchenstruktur auf. So setzt sich das Erzählgerüst aus mehreren autonomen, in sich abgeschlossenen und voneinander isolierten Handlungssträngen ${ }^{16}$ zusammen, die parallel verlaufen, sich verzweigen, wieder voneinander trennen, um schließlich alle einem Erzählhöhepunkt in diesem Fall die Geschichte einer großen Liebe - zuzustreben. Obwohl alle Handlungsstränge einen eigenen, von anderen Erzählelementen isolierten, roten Faden haben, machen sie gerade durch das Hin- und Herpendeln im Romanverlauf und ihr ständiges Ineinandergreifen die Gegensätze zwischen „Gut“ und „Böse“ bewusst, die einander in dauernder Spannung wechselseitig bedingen. So funktionieren etwa „böse“ Figuren, wie Grga Čokolin, der das Glück des Paares Pavle und Dora verhindern will, nach demselben Prinzip wie die böse Stiefmutter in „Schneewittchen“, während die ,gute“ Figur Jerko, den braven Zwergen gleich, die Handlung ständig einem glücklichen Ende zuzuführen bestrebt ist, somit dem Guten zum klaren Triumph über das Böse verhilft. In diesem Sinne dienen nicht nur die Nebenhandlungen und die realistisch kolorierten Episoden, sondern sämtliche von den handelnden Figuren vollbrachte Taten lediglich der Exemplifizierung bzw. der Veranschaulichung und sind dazu angetan, die Tendenz des Großen und Ganzen zu betonen und zu untermauern.

Auch wenn die „Funktionen der Figuren-Akteure“ (vgl. Propp 1975) im romantischen Narrationsparadigma dieses ersten historischen Romans der kroatischen Literatur mit wesentlich anderen Inhalten versehen sind als jene des Märchen, ähneln die flache Darstellung der Akteure und die vereinfachte Kontrastierung der Charaktere sowohl aufgrund der Statik als auch der „Eindimensionalität“ (vgl. Lüthi 1997: 813) des ideellen Gehalts der schablonisierten Darstellung im Märchen. So etwa werden die Helden in Des Goldschmieds Gold als statische Charaktere gezeichnet - als unerschrockene Kämpfer für das Wohl der Nation, denen große Aufgaben auferlegt sind, deren Bewältigung die Überwindung vielfältiger Hindernisse erfordert. ${ }^{17}$ Und obwohl ständig Gefahren ausgesetzt und vor wichtigen, oftmals schicksalhaften Entscheidungen stehend, sind den Protagonisten - nicht nur den Hauptakteuren, sondern auch sämtlichen Nebenfiguren - Zweifel und Unsicherheit vollkommen fremd, sie bleiben, streng nach einer Schablone geordnet und gegliedert, bis zum narrativen

${ }^{16}$ So finden sich als Handlungsstränge etwa der Streit der Zagreber Bürger mit Stjepko Gregorijanec, die Auseinandersetzungen zwischen Pavao und seinem Vater, die geheimen Verbindungen zwischen der verwitweten Klara und Stjepko Gregorijanec, die Intrigen verschiedenster Art von Grga Cokolin, das Schicksal des tauben Jerko, Radaks Geschichte etc.

17 So muss Pavao beispielsweise nicht nur eine Reihe von Hindernissen überwinden, wie etwa den Streit seines Vaters mit den Bürgern von Zagreb, sondern des Öfteren auch das eigene Leben aufs Spiel setzen, um Doras Hand zu gewinnen. Doras Vater, Petar Krupić, muss hingegen nicht nur so manchen Konflikt mit Gregorijanec und seinen Mitbürgern austragen, sondern die eigene Tochter opfern, um Medvedgrad für Zagreb zurückzuerobern. 
Ende unverändert Träger einer bestimmten Charaktereigenschaft. Die abstrakten Figuren sind in der Regel „essenziell bestimmt“ (vgl. Lasić 1965: 181), auf das Wesentliche reduziert und durch klare und unveränderliche, gute oder schlechte Eigenschaften charakterisiert. ${ }^{18}$

Bei den von Šenoa, Tomić, Kumičić und insbesondere den von Kovačić entworfenen Gestalten handelt es sich nun keineswegs um ,papierene“ Märchenfiguren in dem Sinn, dass ihnen ,abgeschnittene Körperteile unverzüglich wieder nachwachsen würden“ (vgl. Lüthi 1997: 14), jedoch steht der dem Körper zugefügte Schmerz nicht für den Schmerz eines konkreten Menschen. Analog zur Fiktionsintention des Märchens dient das Böse in Gestalt von verwerflichen Eigenschaften lediglich dazu, den im Vorherein feststehenden, essenziellen Aufgaben jedes einzelnen Helden noch mehr Gewicht zu verleihen.

Anders als im Volksmärchen oft der Fall, lässt Šenoa seine Figuren die ihnen gestellten Aufgaben nicht um des Abenteuers willen bewältigen. Diese sind vielmehr dazu angetan, dadurch, dass sie soziales Bewusstsein und konkretes politisch-nationales Engagement erfordern, den Aufstieg des menschlichen Geistes bzw. die Rückkehr zu seinem göttlichen Ursprung zu gewährleisten. Im Hinblick darauf fügen sich disparate Elemente leichtfüßig zu einem harmonischen Zusammenspiel mit dem Ziel der Errichtung einer neuen Gesellschaft, die, dem dichterischen Szenarium gemäß, die vollkommene Idylle darstellt. Auch wenn sich Šenoas märchenhafter Diskurs in dieser Hinsicht ganz offensichtlich sowohl an Goethes allegorisch-symbolische als auch an Dickens sozialkritische Märchen anlehnt, kann er mit einiger Berechtigung als eigenständige kroatische Märchenvariante bezeichnet werden.

Zwar lässt sich weder die von Šenoa in Zlatarevo zlato präsentierte Fiktion, noch die Prosawelt irgendeines anderen zeitgenössischen Romanschriftstellers in eine direkte Verbindung mit den von Propp und Lüthi für das Märchen in seiner „reinen Form“ festgelegten Gesetzmäßigkeiten des Märchens bringen - viele davon sind ihnen in programmatisch-deklarativer Hinsicht geradezu entgegengesetzt -, das Wertesystem, die Orientierung hin zu Abstraktheit und Transzendenz, die essenziellstatische Zeichnung der Figuren sowie die dynamische, mit dem Unglaublichen operierende Sujetkonstruktion legen dennoch nahe, diese zwei auf den ersten Blick

${ }_{18}$ Auch wenn der Handlungsbogen über zwanzig Jahre umfasst, bleiben die agierenden Personen von ihrem ersten Auftreten bis zum Schluss nicht hinsichtlich ihrer Charakterisierung, sondern auch altersmäßig unverändert. Dora etwa wird von dem Moment ihrer Einführung in die Handlung bis zur ihrem Tod als wunderschönes sechzehnjähriges Mädchen dargestellt (vgl. Šenoa 1997: 86, 278, 279). Diese unmissverständliche Statik bei der Personendarstellung ist insbesondere bei der Beschreibung von Doras Patin Magda ersichtlich: „Ljudi nisu pamtili, da je kada mlada bila, niti su opazili, da se stari, jednaka, te jednaka kao starinska slika, koja viseć godine i godine u zabitnoj kuli svoga lika ne mijenja.“ (ibid. 84) [Die Menschen erinnerten sich nicht, dass sie einmal jung war, sie merkten überhaupt nicht, dass sie alterte, sie war gleich, immer gleich wie ein altertümliches Bild, das, jahrelang in seinem abgeschiedenen Turm, seine Gestalt nicht ändert.] 
konträren Prosadiskurse analog zu lesen. Durch die Gleichsetzung eines engagierten Literaturkonzepts, das in der konkreten Raumzeitlichkeit einer Nation festgelegt ist, mit dem auf dem Abstrakten und Unglaublichen basierenden Märchenparadigma treten nicht nur die Relativität und Inkonsequenz der explizit bekundeten literarischen Orientierung, sondern auch die Veränderlichkeit und Relativität der kulturellen und stilistischen Konstrukte an die Oberfläche. Ein Herangehen an die dezidiert dem Realismus und dem gesellschaftlichen Engagement verpflichtete kroatische Literatur des 19. Jahrhunderts mit dem Interpretationsinstrumentarium des Märchens macht deutlich, dass sich die Wahrnehmung grundlegender poetologischer Merkmale des Märchens gemäß dessen seit dem 19. Jahrhundert im abendländischen kulturellen Kontext vorliegenden Definition viel eher dem dominanten Kulturparadigma, dem individuellen Horizont des Autors und dem Ausgangspunkt der Interpretation verdankt als dem programmatischen Willen des Autors.

\section{A b k ü r z u n g e $n$}

Barac 1977:

Bausinger 2004:

Biti 2001:

\section{tiger Begriffe, Reinbek bei Hamburg}

Bogatyrev - Jakobson 1972: Petr Bogatyrev, Roman Jakobson, Die Folklore als eine besondere Form des Schaffens, in: Heinz Blumensath (Hg.), Strukturalismus in der Literaturwissenschaft, Köln,13-25

Bošković-Stulli 1975: Maja Bošković-Stulli, Kroatische Volksmärchen, aus dem Kroatischen übersetzt von W. Eschker und V. Milak (= Die Märchen der Weltliteratur), Düsseldorf

Bošković-Stulli 1982: Maja Bošković-Stulli, Bajka, Umjetnost riječi XXVI, 1-2, 113-122

Brackert 1994:

Crnković 1990: $\quad$ Milan Crnović, Dječja književnost. Priručnik za studente i nastavnike, Zagreb

Crnković - Težak 2002: Milan Crnković, Dubravka Težak, Povijest hrvatske dječje književnosti od početaka do 1955. godine, Zagreb

Drndarski 1978: $\quad$ Mirjana Drndarski, Narodna bajka u modernoj književnosti, Beograd

Jolles 1972:

Karlinger 1973:

Katušić 2006:

Kekez 1983:

Klotz 2002:
André Jolles, Einfache Formen, Legende, Sage, Mythe, Rätsel, Spruch, Kasus, Memorabile, Märchen, Witz, 4. Auflage, Tübingen [1930]

Felix Karlinger, Wege der Märchenforschung, Darmstadt

Bernarda Katušić, Bajka u carstvu razlika, in: Dubravka Oraić Tolić, Ernő Kulcsár Szabó (Hg.), Kulturni Stereotipi, Koncepti identiteta u srednjoeuropskim književnostima, Zagreb, 229-253

Josip Kekez, Usmena književnost, in: Zdenko Škreb, Ante Stamać (Hg.), Uvod u književnost, 3. überarbeitete Auflage, Zagreb, 175-253 Volker Klotz, Das europäische Kunstmärchen. Fünfundzwanzig Kapitel seiner Geschichte von der Renaissance bis zur Moderne, 3. Auflage, München 
Lasić 1965:

Lüthi 1997:

Matičević 1997:

Nemec 1994:

Neuhaus 2005:

Petzold 1981:

Propp 1975:

Ranke 1978:

Šenoa 1997:

Sicel 1972:

Sicel 1993:

Solar 2004:

Sutić 1978:

Težak 1997:

Tismar 1981:

Vrkić 1997:

Wührl 2003:

Žmegač 1994:
Stanko Lasić, Roman Šenoina doba (1863-1881), Zagreb

Max Lüthi, Das europäische Volksmärchen. Form und Wesen, Tübingen - Stuttgart [1947]

Ivica Matičević, Hrvatske bajke i basne, Zagreb

Krešimir Nemec, Povijest hrvatskog romana, Zagreb

Stefan Neuhaus, Märchen, Tübingen - Basel

Dieter Petzold, Das englische Kunstmärchen im Neunzehnten Jahrhundert, Tübingen

Vladimir Propp, Morphologie des Märchens, Frankfurt am Main [1928]

Kurt Ranke, Die Welt der Einfachen Formen, Berlin - New York August Šenoa, Zlatarevo zlato, Zagreb

Miroslav Šicel, Programi i manifesti u hrvatskoj književnosti, Zagreb

Miroslav Šicel, Zlatarevo zlato i Prosjak Luka Augusta Šenoe, Zagreb Milivoj Solar, Ideja i priča, Zagreb

Milosav Šutić, Andrićevi „Olujaci“ - izvori i struktura, in: Mirjana Drndarski (Hg.), Narodna bajka u modernoj književnosti, Beograd [1968]

Dubravka Težak, Stjepko Težak, Interpretacija bajke, Zagreb

Jens Tismar, Das deutsche Kunstmärchen des zwanzigsten Jahrhunderts, Stuttgart

Jozo Vrkić, Hrvatske bajke, 8. Auflage, Zagreb [1993]

Paul-Wolfgang Wührl, Das deutsche Kunstmärchen. Geschichte, Botschaft und Erzählstrukturen, 2. Auflage, Baltmannsweiler [1984]

Viktor Žmegač, Märchen, in: Dieter Borchmeyer, Viktor Žmegač (Hg.), Moderne Literatur in Grundbegriffen, Tübingen, 263-268

Bernarda Katušić

Institut für Slawistik der Universität Wien

Universitätscampus $\mathrm{AAKH}$, Hof 3

Spitalgasse 2, 1090 Wien, Österreich

bernarda.katusic@univie.ac.at 